\title{
Gene of the month: FH
}

Roman E Zyla, ${ }^{1}$ Anjelica Hodgson (1) 1,2

1 Laboratory Medicine and Pathobiology, University of Toronto, Toronto, Ontario, Canada

${ }^{2}$ Anatomic Pathology, Toronto General Hospital - University Health Network, Toronto, Ontario, Canada

\section{Correspondence to} Dr Anjelica Hodgson, Anatomic Pathology, Toronto General Hospital - University Health Network, Toronto, ON M5G 2C4, Canada; anjelica. hodgson@uhn.ca

Accepted 22 July 2021

Published Online First 5 August 2021

\section{Check for updates}

(c) Author(s) (or their employer(s)) 2021. No commercial re-use. See rights and permissions. Published by BMJ.

To cite: Zyla RE, Hodgson A. J Clin Pathol 2021:74:615-619.

\begin{abstract}
Fumarate hydratase (FH), encoded by the $F H$ gene, is an enzyme which catalyses the conversion of fumarate to L-malate as part of the tricarboxylic acid cycle. Biallelic germline mutations in $\mathrm{FH}$ result in fumaric aciduria, a metabolic disorder resulting in severe neurological and developmental abnormalities. Heterozygous germline mutations in $\mathrm{FH}$ result in hereditary leiomyomatosis and renal cell carcinoma, a cancer predisposition syndrome. FH deficiency has multiple oncogenic mechanisms including through promotion of aerobic glycolysis, induction of pseudohypoxia, post-translational protein modification and impairment of DNA damage repair by homologous recombination. FH-deficient neoplasms can present with characteristic morphological features that raise suspicion for $\mathrm{FH}$ alterations and also frequently demonstrate loss of $\mathrm{FH}$ immunoreactivity and intracellular accumulation of 2-succinocysteine, also detected by immunohistochemistry.
\end{abstract}

\section{INTRODUCTION}

The $F H$ gene encodes for the protein fumarate hydratase (FH), a key enzyme in the tricarboxylic acid (TCA) cycle. ${ }^{1}$ Pathogenic $F H$ mutations and the resultant deficiency in $\mathrm{FH}$ function result in a myriad of metabolic and cell signalling alterations, many of which promote oncogenesis, which explains the association between germline mutations in $\mathrm{FH}$ and the increased risk for certain neoplasms.

\section{GENE AND PROTEIN STRUCTURE}

$\mathrm{FH}$ is located on chromosome 1 (1q42.3-q43) and is composed of 10 exons. ${ }^{12}$ The $\mathrm{FH}$ protein is a 510-amino acid structure with three key domains: a $\mathrm{N}$-terminal lyase 1 domain, a C-terminal fumarase $\mathrm{C}$ domain and a central domain which interacts with other FH monomers. ${ }^{13}$ In its functional form, FH exists as a homotetramer ${ }^{4}$ and can be localised in the mitochondria or the cytosol. Both mitochondrial and cytosolic FH are encoded by nuclear DNA, with the differential localisation mediated by alternative transcription products, one of which produces a precursor protein with an $\mathrm{N}$-terminal mitochondrial localisation signal. ${ }^{15}$

\section{PHYSIOLOGICAL ROLE}

Within the mitochondria, homotetrameric $\mathrm{FH}$ catalyses the reversible conversion of fumarate to L-malate as part of the TCA cycle, a critical pathway in cellular metabolism which processes substrates for the production of ATP through oxidative phosphorylation. $^{14}$

Cytosolic FH can be translocated to the nucleus to participate in DNA damage repair. ${ }^{6}$ Upon localisation to the nucleus, FH catalyses fumarate production, with the resultant increased fumarate concentration inhibiting the histone demethylase KDM2B and promoting repair of double-strand breaks by non-homologous end-joining. ${ }^{67}$ In addition, cytosolic FH is crucial for proper functioning of the urea cycle. ${ }^{8}$ In cases of FH deficiency, excess cytosolic fumarate leads to accumulation of argininosuccinate, and FH-deficient cells become acutely dependent on exogenous arginine due to disruption of normal urea cycle metabolism. ${ }^{18}$

\section{ROLE IN DISEASE}

\section{Fumaric aciduria}

Biallelic germline pathogenic mutations in $\mathrm{FH}$ are very rare and result in fumaric aciduria, a condition that occurs due to a systemic inability to effectively convert fumarate to malate. ${ }^{9}$ Affected patients have high levels of urinary fumarate and detectable aberrations in serum levels of other TCA cycle metabolites. ${ }^{310}$ The associated phenotype is severe with multiple dysmorphic features, hypotonia and profound, global developmental deficits presenting in infancy; only rare survival past childhood has been reported. ${ }^{310}$

\section{FH deficiency and tumour development}

Putatively oncogenic missense mutations are most common in exons 4, 5, 7 and $8 .{ }^{11} \mathrm{FH}$-deficient cells lose the capacity to metabolise fumarate and intracellular levels consequently increase, resulting in a series of compensatory metabolic shifts. ${ }^{1}$ For example, mitochondrial oxidative phosphorylation is reduced while aerobic glycolysis, glutamine catabolism and major anabolic pathways are upregulated; these are features of the Warburg effect, one of the metabolic hallmarks of malignancy. ${ }^{1} 1213$

In addition to its impact on TCA cycle dysregulation, increased intracellular fumarate is capable of inducing other oncogenic cellular alterations, justifying its classification as an oncometabolite. ${ }^{14}$ One of the major changes associated with increased fumarate is constitutive stabilisation of hypoxia inducible factor (HIF) $-1 \alpha$ and HIF-2 $\alpha .{ }^{15}$ Under normal physiological conditions, HIF- $1 \alpha$ and HIF- $2 \alpha$ are constitutively hydroxylated by a prolyl hydroxylase, which results in their downstream ubiquitylation and subsequent proteasomal degradation. ${ }^{16}$ Under hypoxic conditions, these proteins are stabilised and capable of inducing transcription of key genes involved in angiogenesis and cell growth. ${ }^{17}$ Elevated intracellular fumarate is believed to inhibit $\alpha$-ketoglutarate-dependent dioxygenases including prolyl hydroxylase and thereby prevent ubiquitylation and degradation of these 


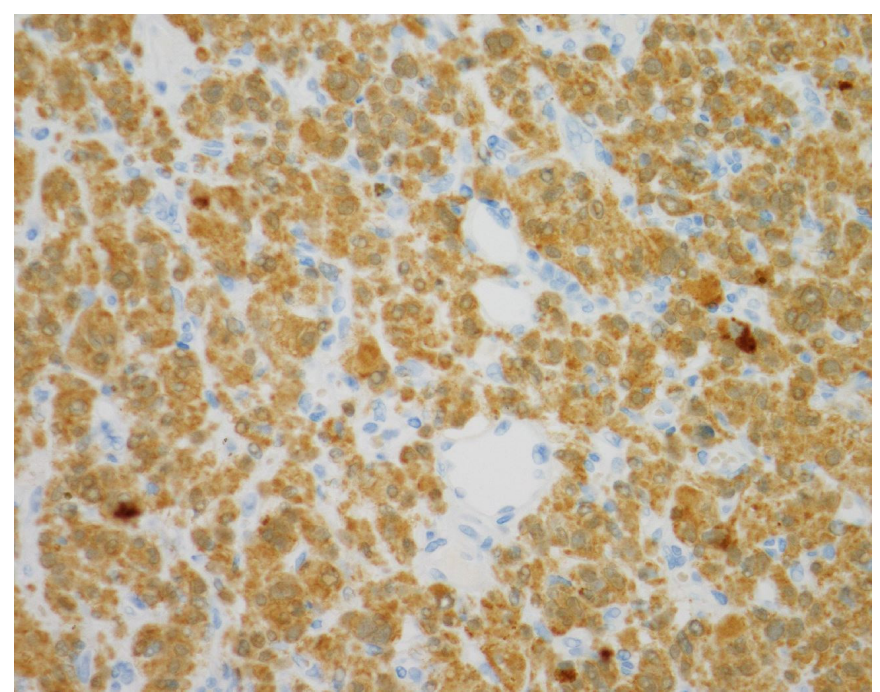

Figure 1 2-succinocysteine (2-SC) immunohistochemistry. Increased intracellular fumarate levels as a result of fumarate hydratase deficiency leads to increased protein succination which can be detected through immunohistochemistry for 2-SC. In this example of a fumarate hydratase-deficient uterine leiomyoma, the tumour cells show abnormal diffuse cytoplasmic granular expression of 2-SC, in contrast to background endothelial, stromal and inflammatory cells which show no expression. Image magnification $\times 400$.

HIF-family proteins. ${ }^{15} 161819$ Moreover, FH deficiency is associated with increased reactive oxygen species which can also inhibit prolyl hydroxylase activity. ${ }^{18}$ Additional evidence for the importance of this signalling pathway has been found in FH-deficient uterine leiomyomata (discussed below), which have higher microvessel density and increased expression of vascular endothelial growth factor than their
non-FH-deficient counterparts. ${ }^{20}$ However, the relative contribution of these mechanisms to HIF-family activity and its contribution to oncogenesis in $\mathrm{FH}$-deficient cells remains a matter of controversy. ${ }^{15}$

Increased fumarate levels also result in post-translational succination of cysteine residues on a number of different proteins. One of these proteins is KEAP1 which normally induces degradation of NRF2. ${ }^{15}$ NRF2 upregulation leads to increased intracellular ferritin and the resultant activation of the transcription factor FOXM1 which promotes cellular proliferation as well as an antioxidant response phenotype. ${ }^{1} 1521$ Other proteins preferentially succinated in $\mathrm{FH}$-deficient cells include those involved in metabolism and the SWItch/Sucrose Non-Fermentable (SWI/SNF) chromatin remodelling complex. ${ }^{14}$ Positive immunohistochemical staining for 2-succinocysteine (2-SC) is a marker of protein succination, and has been shown to be a reliable marker for tumourous FH deficiency (figure 1). ${ }^{22}$

In addition, dysregulation of normal $\mathrm{FH}$ activity has been shown to impair DNA repair by homologous recombination through alterations in histone methylation. ${ }^{23}$ An additional more recently described oncogenic effect of $\mathrm{FH}$ deficiency is the promotion of epithelial-to-mesenchymal transition (EMT) via downregulation of the miR-200 family of microRNAs and subsequent upregulation of an EMT-associated signature. $^{24}$

The main consequences of FH deficiency and increased fumarate levels are shown in figure 2.

\section{HEREDITARY LEIOMYOMATOSIS AND RENAL CELL CARCINOMA}

Heterozygous, pathogenic germline mutations in $F H$ are associated with an autosomal dominant cancer predisposition syndrome called hereditary leiomyomatosis and renal

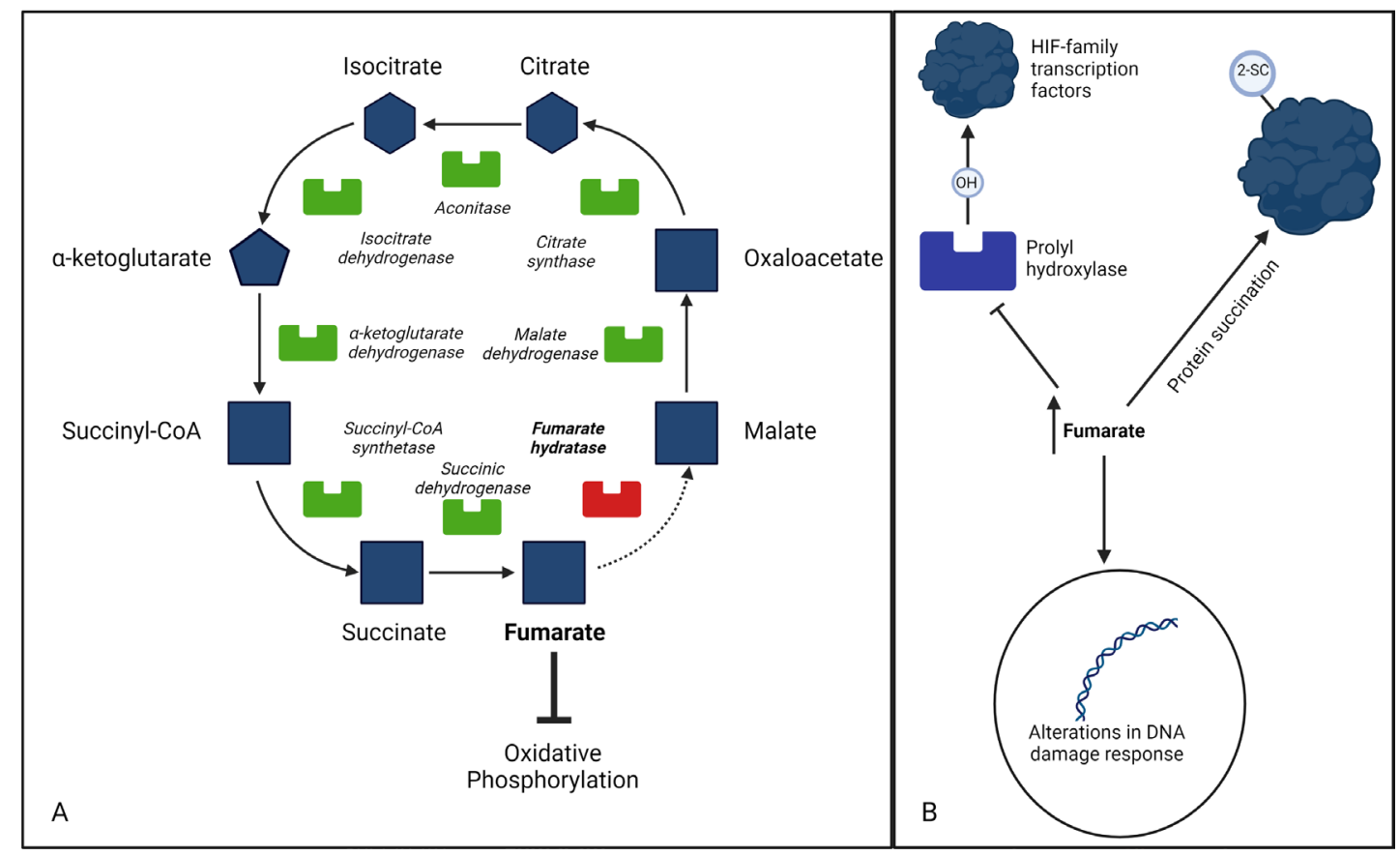

Figure 2 Consequences of increased fumarate levels. Fumarate hydratase catalyses the conversion of fumarate to malate in the tricarboxylic acid cycle (A). Inhibition of this enzyme causes increased levels of fumarate which inhibit oxidative phosphorylation in the mitochondria. Separately, increased levels of cytosolic and nuclear fumarate result in upregulation of HIF-family transcription factors, protein succination and aberrant DNA repair, among other impacts (B). Created with BioRender.com. 2-SC, 2-succinocysteine; HIF, hypoxia inducible factor. 


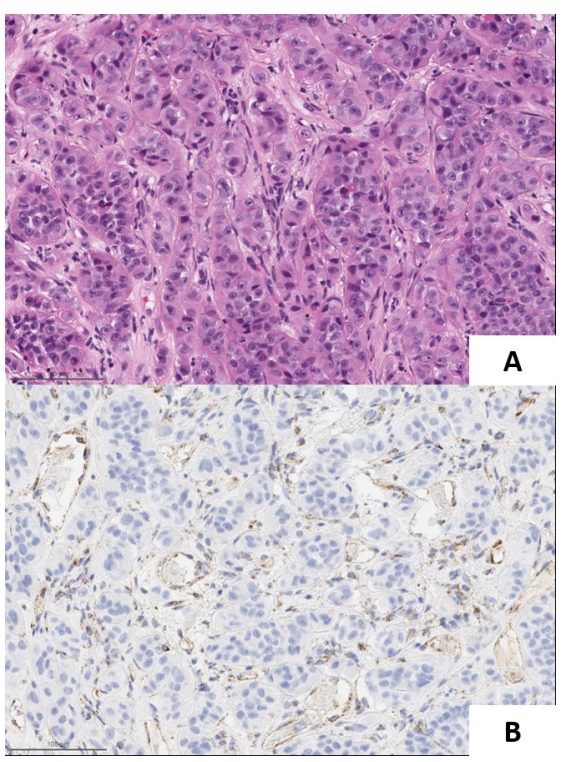

Figure 3 Fumarate hydratase (FH)-deficient renal cell carcinoma. This example shows nested and solid architecture, abundant eosinophilic cytoplasm and conspicuous single macronucleoli (A). Loss of FH immunoexpression can be a useful ancillary technique for confirming this diagnosis; note how background endothelial cells act as an internal control (B). Image magnification $\times 200 x$.

cell carcinoma (HLRCC). ${ }^{25}$ This condition is associated with frequent and multiple cutaneous leiomyomata and aggressive RCCs and, in females, with early-onset, symptomatic uterine leiomyomata. ${ }^{25}$ In keeping with the role of $\mathrm{FH}$ as a tumour suppressor, all somatic tissues in HLRCC patients have only one functional copy of the $\mathrm{FH}$ gene; mutation or loss of this remaining copy results in the 'second hit' that initiates tumourigenesis. ${ }^{15}$ Pathogenic $\mathrm{FH}$ mutation can be reliably detected in up to $100 \%$ of patients meeting clinical criteria for the syndrome; large exonic deletions, which may require multiplex ligationdependent probe amplification to detect, appear to occur only rarely in patients with HLRCC. ${ }^{2526}$ There is evidence to suggest that truncating mutations may be associated with a milder phenotype than missense mutations with the latter postulated to have a dominant-negative impact that actively inhibits homotetramer formation in at least some cases. ${ }^{27}$

\section{FH-deficient neoplasms}

\section{FH-deficient RCC}

RCCs with FH deficiency occur almost exclusively in the setting of HLRCC however, FH-deficient RCC can also arise sporadically secondary to loss of function in both $\mathrm{FH}$ alleles. ${ }^{28-30} \mathrm{FH}$-deficient RCC arises in approximately 15\%-30\% of patients with HLRCC and in the syndromic setting, these tumours are associated with an early age of onset and an aggressive clinical course with one study finding that $50 \%$ of patients died of disease after a median follow-up of only 16 months. ${ }^{25} 3132$

Histologically, the tumours frequently display multiple architectural patterns within the same lesion, with papillary architecture the most common but by no means ubiquitous. ${ }^{31}$ Also characteristic are large, eosinophilic, 'inclusion-like' nucleoli, though their presence is often variable ${ }^{31}$ (figure 3 ). Less common morphological variants include the characteristic low-grade, oncocytic cytology more typically associated with succinate dehydrogenase (SDH)-deficient RCC, and tubulocystic architecture with poorly differentiated foci. ${ }^{33}$ Immunoreactivity for

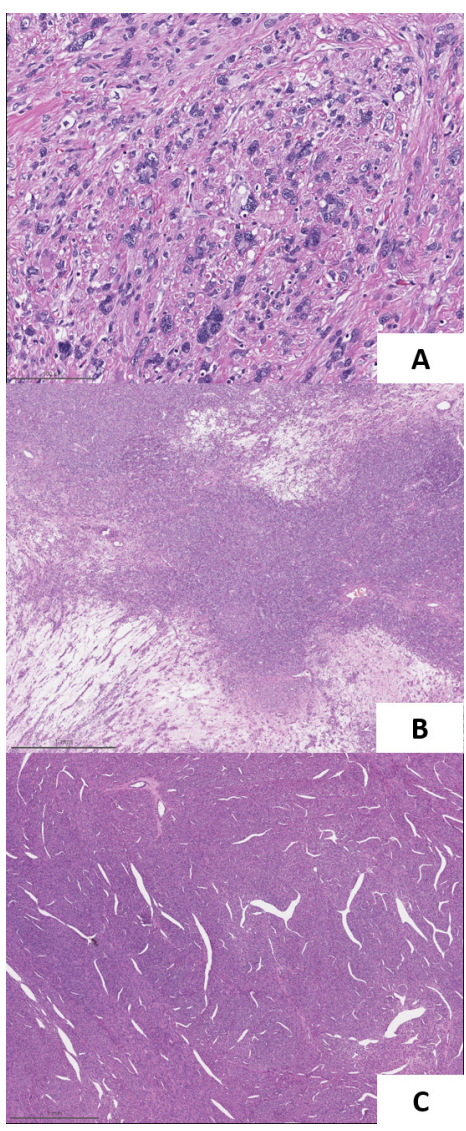

Figure 4 Fumarate hydratase (FH)-deficient uterine leiomyoma. Characteristic features of FH-deficient uterine leiomyomata include marked nuclear atypia, single prominent nucleoli with perinucleolar clearing, hyaline globules (A), 'alveolar-type' stromal oedema (B) and hemangiopericytomatous ('staghorn') blood vessels (C). These morphological features may be seen in tumours with somatic $\mathrm{FH}$ alterations or in the context of germline $\mathrm{FH}$ changes. Image magnification $\times 200(A)$ and $\times 20(B, C)$.

$\mathrm{FH}$ is frequently lost but can be retained in a subset of cases in which a non-functional protein is expressed, while immunohistochemistry for 2-SC can also be a useful ancillary study. ${ }^{28313235}$

Historically, these FH-deficient RCCs were classified as type 2 papillary RCCs, given their nuclear features and frequent papillary architecture. ${ }^{25}$ In The Cancer Genome Atlas Research Network study of papillary RCC, FH-deficient cases clustered with the $\mathrm{CpG}$ island methylator phenotype subgroup of tumours, characterised by multiple metabolic alterations and an aggressive clinical course. ${ }^{36}$ However, HLRCC-associated RCC is now a distinct subtype of RCC in its own right, a reflection of the unique aetiology and clinicopathological characteristics associated with this entity. ${ }^{28} 29$

\section{Uterine leiomyomata}

$\mathrm{FH}$ deficiency is a rare finding in unselected populations of patients with uterine leiomyomata and unlike FH-deficient RCCs, a higher proportion of these $\mathrm{FH}$-deficient uterine tumours are not associated with underlying germline mutation. ${ }^{37-39}$ In the setting of HLRCC, affected women characteristically develop early-onset and symptomatic uterine leiomyomata which often require early surgical intervention. ${ }^{35} 37$

Similar to FH-deficient RCCs, FH-deficient uterine leiomyomata are associated with certain morphological features 
that may raise suspicion as to their underlying pathogenesis. $^{40} \mathrm{FH}$-deficient leiomyomata are more likely than other leiomyomata to present with symplastic-type nuclear atypia, misinterpretation of which may result in erroneous classification as leiomyosarcoma. ${ }^{373841}$ More specific features include hemangiopericytoma-like ('staghorn') blood vessels, 'alveolartype' stromal oedema, eosinophilic cytoplasmic globules and large, eosinophilic nucleoli (figure 4); none of these features in isolation show perfect diagnostic accuracy, but their conspicuous presence within a tumour should prompt further consideration that one is dealing with an FH-deficient tumour, particularly in a young patient. ${ }^{35}$ As with FH-deficient RCC, immunohistochemistry for $\mathrm{FH}$ and 2-SC can be helpful ancillary tools for diagnosis although of note, morphology and immunohistochemistry alone cannot distinguish between a uterine tumour with somatic versus germline $F H$ mutation. As such, consideration should be given to germline testing when characteristic morphology is identified, even in the absence of supportive immunohistochemical results, as it has been shown that neither FH or 2-SC immunohistochemistry correlates perfectly with molecular testing for $\mathrm{FH}$ mutation. ${ }^{35} 42$

FH-deficient leiomyomata of the uterus are not specifically associated with an increased risk of malignancy, however, their identification is extremely important as by recognising the characteristic phenotype and/or confirming FH-deficiency by IHC or molecular testing, pathologists can recommend germline testing for $\mathrm{FH}$ mutations so as to identify patients that may benefit from routine screening for HLRCC-associated RCC. ${ }^{40}$

\section{Cutaneous leiomyomata}

Cutaneous leiomyomata, believed to derive from the arrector pili muscles in hair-bearing skin, are not always associated with FH deficiency and occur outside of the syndromic setting however, when multiple and widespread, are often the most conspicuous manifestation of HLRCC. ${ }^{27}$ In the syndromic setting, affected patients often have multiple grossly apparent papules or nodules ${ }^{27} 43$ and of note, they occur with higher penetrance in men. ${ }^{27}$ Morphologically, cutaneous leiomyomata resemble leiomyomata of other locations and, unlike HLRCC-associated RCCs and uterine leiomyomata, do not have any known distinctive histological features. ${ }^{44} 45$ While benign, these lesions can cause significant morbidity and can be painful, particularly with changes in temperature. ${ }^{43}$ Like FH-deficient uterine leiomyomata, the presence of multiple cutaneous leiomyomata should alert clinicians to test for germline $\mathrm{FH}$ mutation, so that appropriate screening for RCC can be initiated. ${ }^{44}$ Immunohistochemistry for 2-SC can be a useful adjunct for detection of $\mathrm{FH}$-deficiency in these tumours, with the utility of immunohistochemistry for FH considered to be more controversial. ${ }^{44} 45$

\section{Other}

FH-deficiency is associated with some of the same downstream effects on HIF- $1 \alpha$ signalling as $S D H$ family mutations and while SDH deficiency is associated with a different spectrum of tumour predispositions compared with HLRCC, a small amount of overlap does exist. ${ }^{15}$ For example, FH deficiency as a result of germline mutations has been implicated as a rare cause of malignant paragangliomas and pheochromocytomas. ${ }^{46}$ Other FH-deficient tumours include Leydig cell tumours of the testis and rare bladder and breast carcinomas that have been identified in patients with HLRCC. ${ }^{27} 47$ Uterine leiomyosarcoma has been controversially associated with HLRCC, with a study out of
Take home messages

- Fumarate hydratase $(\mathrm{FH})$, encoded by the $\mathrm{FH}$ gene of chromosome 1, catalyses the conversion of fumarate to Lmalate as part of the tricarboxylic acid cycle, and also plays roles in the urea cycle and DNA repair.

- Biallelic germline mutations in $F H$ result in a severe inborn disorder of metabolism, fumaric aciduria, that is characterised by profound neurological and developmental deficits.

- Heterozygous germline loss-of-function mutations in $F H$ cause hereditary leiomyomatosis and renal cell carcinoma, which predisposes to an aggressive variant of renal cell carcinoma and other tumours.

- Some FH-deficient neoplasms have distinct morphological features but immunohistochemistry and/or molecular testing are often required to secure a definitive diagnosis.

Finland reporting a substantially increased risk in these patients, but other large cohort studies have failed to identify any FH-deficient leiomyosarcomas. ${ }^{373848}$

Handling editor Runjan Chetty.

Contributors REZ and AH worked cooperatively and contributed equally to this manuscript.

Funding The authors have not declared a specific grant for this research from any funding agency in the public, commercial or not-for-profit sectors.

Competing interests None declared.

Patient consent for publication Not required.

Provenance and peer review Commissioned; internally peer reviewed.

\section{ORCID iD}

Anjelica Hodgson http://orcid.org/0000-0002-4069-2000

\section{REFERENCES}

1 Schmidt C, Sciacovelli M, Frezza C. Fumarate hydratase in cancer: a multifaceted tumour suppressor. Semin Cell Dev Biol 2020;98:15-25.

2 Tomlinson IPM, Alam NA, Rowan AJ, et al. Germline mutations in FH predispose to dominantly inherited uterine fibroids, skin leiomyomata and papillary renal cell cancer. Nat Genet 2002:30:406-10

3 Allegri G, Fernandes MJ, Scalco FB, et al. Fumaric aciduria: an overview and the first Brazilian case report. J Inherit Metab Dis 2010;33:411-9.

4 Ajalla Aleixo MA, Rangel VL, Rustiguel JK, et al. Structural, biochemical and biophysical characterization of recombinant human fumarate hydratase. Febs $J$ 2019;286:1925-40.

5 Dik E, Naamati A, Asraf $H$, et al. Human fumarate hydratase is dual localized by an alternative transcription initiation mechanism. Traffic 2016;17:720-32.

6 Yogev 0 , Yogev 0 , Singer $E$, et al. Fumarase: a mitochondrial metabolic enzyme and a cytosolic/nuclear component of the DNA damage response. PLOS Biol 2010;8:e1000328.

7 Jiang Y, Qian X, Shen J, et al. Local generation of fumarate promotes DNA repair through inhibition of histone $\mathrm{H} 3$ demethylation. Nat Cell Biol 2015;17:1158-68.

8 Adam J, Yang M, Bauerschmidt C, et al. A role for cytosolic fumarate hydratase in urea cycle metabolism and renal neoplasia. Cell Rep 2013;3:1440-8.

9 Zinn AB, Kerr DS, Hoppel CL. Fumarase deficiency: a new cause of mitochondrial encephalomyopathy. N Engl J Med 1986;315:469-75.

10 Kerrigan JF, Aleck KA, Tarby TJ, et al. Fumaric aciduria: clinical and imaging features. Ann Neurol 2000;47:583-8.

11 Bayley J-P, Launonen V, Tomlinson IPM. The FH mutation database: an online database of fumarate hydratase mutations involved in the MCUL (HLRCC) tumor syndrome and congenital fumarase deficiency. BMC Med Genet 2008;9:20.

12 Tyrakis PA, Yurkovich ME, Sciacovelli M, et al. Fumarate hydratase loss causes combined respiratory chain defects. Cell Rep 2017;21:1036-47.

13 Tong W-H, Sourbier C, Kovtunovych G, et al. The glycolytic shift in fumarate-hydratasedeficient kidney cancer lowers AMPK levels, increases anabolic propensities and lowers cellular iron levels. Cancer Cell 2011;20:315-27.

14 Kulkarni RA, Bak DW, Wei D, et al. A chemoproteomic portrait of the oncometabolite fumarate. Nat Chem Biol 2019;15:391-400.

15 Ooi A. Advances in hereditary leiomyomatosis and renal cell carcinoma (HLRCC) research. Semin Cancer Biol 2020;61:158-66. 
16 Isaacs JS, Jung YJ, Mole DR, et al. HIF overexpression correlates with biallelic loss of fumarate hydratase in renal cancer: novel role of fumarate in regulation of HIF stability. Cancer Cell 2005;8:143-53.

17 Ooi A, Wong J-C, Petillo D, et al. An antioxidant response phenotype shared between hereditary and sporadic type 2 papillary renal cell carcinoma. Cancer Cell 2011;20:511-23.

18 Sudarshan S, Sourbier C, Kong H-S, et al. Fumarate hydratase deficiency in renal cancer induces glycolytic addiction and hypoxia-inducible transcription factor 1alpha stabilization by glucose-dependent generation of reactive oxygen species. Mol Cell Biol 2009;29:4080-90.

19 Xiao M, Yang $H$, Xu W, et al. Inhibition of $\alpha-K G$-dependent histone and DNA demethylases by fumarate and succinate that are accumulated in mutations of $\mathrm{FH}$ and SDH tumor suppressors. Genes Dev 2012;26:1326-38.

20 Pollard P, Wortham N, Barclay E, et al. Evidence of increased microvessel density and activation of the hypoxia pathway in tumours from the hereditary leiomyomatosis and renal cell cancer syndrome. J Pathol 2005;205:41-9.

21 Kerins MJ, Vashisht AA, Liang BX-T, et al. Fumarate mediates a chronic proliferative signal in fumarate hydratase-inactivated cancer cells by increasing transcription and translation of ferritin genes. Mol Cell Biol 2017;37:e00079-17.

22 Bardella C, El-Bahrawy M, Frizzell N, et al. Aberrant succination of proteins in fumarate hydratase-deficient mice and HLRCC patients is a robust biomarker of mutation status. J Pathol 2011;225:4-11.

23 Sulkowski PL, Sundaram RK, Oeck S, et al. Krebs-cycle-deficient hereditary cancer syndromes are defined by defects in homologous-recombination DNA repair. Nat Genet 2018;50:1086-92.

24 Sciacovelli $\mathrm{M}$, Gonçalves $\mathrm{E}$, Johnson $\mathrm{TI}$, et al. Fumarate is an epigenetic modifier that elicits epithelial-to-mesenchymal transition. Nature 2016;537:544-7.

25 Menko FH, Maher ER, Schmidt LS, et al. Hereditary leiomyomatosis and renal cell cancer (HLRCC): renal cancer risk, surveillance and treatment. Fam Cancer 2014;13:637-44.

26 Ahvenainen $\mathrm{T}$, Lehtonen $\mathrm{HJ}$, Lehtonen $\mathrm{R}$, et al. Mutation screening of fumarate hydratase by multiplex ligation-dependent probe amplification: detection of exonic deletion in a patient with leiomyomatosis and renal cell cancer. Cancer Genet Cytogenet 2008; 183:83-8.

27 Lehtonen HJ. Hereditary leiomyomatosis and renal cell cancer: update on clinical and molecular characteristics. Fam Cancer 2011;10:397-411.

28 Gleeson JP, Nikolovski I, Dinatale R, et al. Comprehensive molecular characterization and response to therapy in fumarate hydratase-deficient renal cell carcinoma. Clin Cancer Res 2021:27:2910-9.

29 Trpkov K, Hes O. New and emerging renal entities: a perspective post-WHO 2016 classification. Histopathology 2019;74:31-59.

30 Trpkov K, Hes 0, Agaimy A, et al. Fumarate hydratase-deficient renal cell carcinoma is strongly correlated with fumarate hydratase mutation and hereditary leiomyomatosis and renal cell carcinoma syndrome. Am J Surg Pathol 2016;40:865-75.

31 Lau HD, Chan E, Fan AC, et al. A clinicopathologic and molecular analysis of fumarate hydratase-deficient renal cell carcinoma in 32 patients. Am J Surg Pathol 2020;44:98-110.

32 Chen Y-B, Brannon AR, Toubaji A, et al. Hereditary leiomyomatosis and renal cell carcinoma syndrome-associated renal cancer: recognition of the syndrome by pathologic features and the utility of detecting aberrant succination by immunohistochemistry. Am J Surg Pathol 2014;38:627-37.
33 Smith SC, Sirohi D, Ohe C, et al. A distinctive, low-grade oncocytic fumarate hydratase-deficient renal cell carcinoma, morphologically reminiscent of succinate dehydrogenase-deficient renal cell carcinoma. Histopathology 2017;71:42-52.

34 Smith SC, Trpkov K, Chen Y-B, et al. Tubulocystic carcinoma of the kidney with poorly differentiated foci: a frequent morphologic pattern of fumarate hydratase-deficient renal cell carcinoma. Am J Surg Pathol 2016;40:1457-72.

35 Chan E, Rabban JT, Mak J, et al. Detailed morphologic and immunohistochemical characterization of myomectomy and hysterectomy specimens from women with hereditary leiomyomatosis and renal cell carcinoma syndrome (HLRCC). Am J Surg Pathol 2019;43:1170-9.

36 Cancer Genome Atlas Research Network, Linehan WM, Spellman PT, et al. Comprehensive molecular characterization of papillary renal-cell carcinoma. N Eng/ J Med 2016:374:135-45.

37 Harrison WJ, Andrici J, Maclean F, et al. Fumarate hydratase-deficient uterine leiomyomas occur in both the syndromic and sporadic settings. Am J Surg Pathol 2016;40:599-607.

38 Miettinen M, Felisiak-Golabek A, Wasag B, et al. Fumarase-deficient uterine leiomyomas: an immunohistochemical, molecular genetic, and clinicopathologic study of 86 cases. Am J Surg Pathol 2016;40:1661-9.

39 Siegler L, Erber R, Burghaus S, et al. Fumarate hydratase (FH) deficiency in uterine leiomyomas: recognition by histological features versus blind immunoscreening. Virchows Arch 2018;472:789-96.

40 Garg K, Rabban J. Hereditary leiomyomatosis and renal cell carcinoma syndrome associated uterine smooth muscle tumors: bridging morphology and clinical screening. Genes Chromosomes Cancer 2021;60:210-6.

41 Zhang Q, Poropatich K, Ubago J, et al. Fumarate hydratase mutations and alterations in leiomyoma with bizarre nuclei. Int J Gynecol Pathol 2018;37:421-30.

42 Joseph NM, Solomon DA, Frizzell N, et al. Morphology and immunohistochemistry for $2 \mathrm{SC}$ and $\mathrm{FH}$ aid in detection of fumarate hydratase gene aberrations in uterine leiomyomas from young patients. Am J Surg Pathol 2015;39:1529-39.

43 Young KZ, Raisanen TD, Else T, et al. A few pink papules in an adult woman: incidenta finding leads to diagnosis of hereditary leiomyomatosis and renal cell cancer. JAAD Case Reports 2019:5:419-21.

44 Carter CS, Skala SL, Chinnaiyan AM, et al. Immunohistochemical characterization of fumarate hydratase (FH) and succinate dehydrogenase (SDH) in cutaneous leiomyomas for detection of familial cancer syndromes. Am J Surg Pathol 2017:41:801-9.

45 Buelow B, Cohen J, Nagymanyoki Z, et al. Immunohistochemistry for 2-succinocysteine (2SC) and fumarate hydratase (FH) in cutaneous leiomyomas may aid in identification of patients with HLRCC (hereditary leiomyomatosis and renal cell carcinoma syndrome). Am J Surg Pathol 2016;40:982-8.

46 Castro-Vega LJ, Buffet A, De Cubas AA, et al. Germline mutations in FH confer predisposition to malignant pheochromocytomas and paragangliomas. Hum $\mathrm{Mol}$ Genet 2014;23:2440-6.

47 Carvajal-Carmona LG, Alam NA, Pollard PJ, et al. Adult Leydig cell tumors of the testis caused by germline fumarate hydratase mutations. J Clin Endocrinol Metab 2006;91:3071-5.

48 Lehtonen HJ, Kiuru M, Ylisaukko-Oja SK, et al. Increased risk of cancer in patients with fumarate hydratase germline mutation. J Med Genet 2006;43:523-6. 